\title{
Role of LOX-1 in Ang II-induced oxidative functional damage in renal tubular epithelial cells
}

\author{
YANFANG XU ${ }^{1}$, SHIWEI RUAN ${ }^{1}$, HONG XIE ${ }^{2}$ and JIUMAO LIN ${ }^{1}$ \\ ${ }^{1}$ Academy of Integrative Medicine, Fujian University of Traditional Chinese Medicine, Fuzhou 350108; \\ ${ }^{2}$ Department of Cardiology, First Affiliated Hospital, Fujian Medical University, Fuzhou 350005, P.R. China
}

Received June 7, 2010; Accepted August 2, 2010

DOI: 10.3892/ijmm_00000514

\begin{abstract}
The lectin-like oxidized low-density lipoprotein receptor-1 (LOX-1), plays an important role in angiotension II (Ang II)-induced hypertensive renal injury associated with pro-inflammatory responses, tubular functional damage and cellular apotosis. In this study, we report on the role of LOX-1 in Ang II-induced oxidative functional damage and underlying signaling in human renal proximal tubular epithelial cells (HRPTEpiCs). The exposure to Ang II enhanced the expression of the NADPH oxidases (the p22phox, p47phox and Nox4 subunits), LOX-1 and the adhesion molecule, ICAM-1. It also promoted monocytic U937 cell adherences to HRPTEpiCs, increased reactive oxygen species formation and stimulated apotosis, which was concomitant with an increase in the activation of p38 and p44/42 mitogen-activated protein kinases (MAPK). Furthermore, the Ang II treatment disturbed the balance of the Bcl-2 family proteins, destabilized mitochondrial membrane potential, and subsequently triggered the release of cytochrome $\mathrm{c}$ into the cytosol, causing the activation of caspase-3. The NADPH oxidase inhibitors and LOX-1 small interfering RNA markedly ameliorated these detrimental effects by reducing LOX-1 expression and MAPK activation. The p38 and p44/42MAPK inhibitors also inhibited the Ang II-induced functional damage without affecting LOX-1 expression in the HRPTEpiCs. These observations suggest that LOX-1 mediates Ang II-induced renal tubular epithelial dysfunction. In addition, MAPK pathway activation occurs downstream of the Ang II/reactive oxygen species/ LOX-1 cascade.
\end{abstract}

\section{Introduction}

A growing body of evidence indicates that the lectin-like oxidized low-density lipoprotein (LDL) receptor-1 (LOX-1)

Correspondence to: Dr Yanfang Xu, Academy of Integrative Medicine, Fujian University of Traditional Chinese Medicine, Huatuo Road 1, Shangjie, Minhou, Fuzhou 350108, P.R. China E-mail: xuyanfang99@hotmail.com

Key words: oxidized low-density lipoprotein receptors, adhesion molecule, apoptosis, reactive oxygen species plays an important role in the pathophysiology of renal diseases. LOX-1 is strongly expressed in the kidneys of saltloaded Dahl salt-sensitive, obese diabetic and chronic renal failure rats. Additionally, LOX-1 expression parallels renal dysfunction, suggesting a possible link between LOX-1 and the progression of renal failure (1-3). Dominguez et al have shown that obese rats, a model of metabolic syndrome in which hypertension is the dominant feature, present with renal enlargement, intense oxidative stress, lipid accumulation, leukocyte infiltration, as well as renal dysfunction and fibrosis. These rats also showed LOX-1 overexpression, and treatment with anti-LOX-1 antibody significantly limited or prevented the detrimental effects of obesity on renal function (4). In vitro, the exposure of normal rat proximal tubules to oxidized LDL (oxLDL) has been shown to markedly increase levels of LOX-1. In addition, oxLDL exposure, changed their quiescent epithelial phenotype into a pro-inflammatory one, which is characterized by reduced E-cadherin expression, greater epithelial permeability, increased ICAM-1 expression, enhanced leukocyte adherence and apoptosis (2). The action of oxLDL was partially blocked by an anti-LOX-1 antibody, indicating that oxLDL activity is dependent on LOX-1. On the whole, these observations suggest that the up-regulation of LOX-1 promotes apoptosis and the production of inflammatory and profibrotic mediators in tubular epithelial cells, culminating in inflammation, tubular atrophy, interstitial fibrosis.

The renin-angiotensin system and its effector hormone, angiotensin II (Ang II), have well-known endocrine properties that contribute to renal fibrosis. In addition to its vasoactive and hemodynamic effects, Ang II also acts directly as a growth factor and pro-inflammatory cytokine (5). Previous studies have shown that Ang II, through the activation of Ang II receptor type 1 (AT1), stimulates LOX-1 expression on the transcriptional level, and that an active NF- $\mathrm{BB}$ binding site is necessary for this induction (6). In turn, LOX-1 activation up-regulates AT1 receptor expression (7). The activation of both the AT1 and LOX-1 receptors induces a state of oxidative stress, resulting in the production of superoxide anion. Superoxide anion serves as a signaling molecule, mediating the activity of $\mathrm{NF}-\mathrm{\kappa B}$, which co-ordinates the upregulation of pro-inflammatory gene expression including LOX-1, monocyte chemotactic protein-1 and ICAM- 1 . These conditions enhance oxidative stress, alter cell function and activate apoptosis, promoting the development of renal fibrosis 
and damage $(6,8,9)$. A recent study has indicated that the targeted deletion of LOX-1 reduces Ang II-induced hypertensive renal injury, suggesting that there is cross-talk between oxidative stress, LOX-1 and Ang II in the genesis of hypertension and renal injury (10). In order to gain further insight into the effects of LOX-1 on Ang II-mediated renal injury, we conducted the present study examining the effects of Ang II activation on LOX-1 expression and the role of LOX-1 in Ang II-induced oxidative functional damage in human renal proximal tubular epithelial cells (HRPTEpiCs).

\section{Materials and methods}

Cell culture. All experiments were done using HRPTEpiCs (ScienCell, San Diego, CA, USA) isolated from human renal tissue. The HRPTEpiCs were cultured in epithelial cell medium (EpiCM; ScienCell) supplemented with 2\% fetal bovine serum (FBS). U937 cells, originally derived from human histiocytic lymphoma, were obtained from the American Type Culture Collection (Rockville, MD, USA). The U937 cells were grown in suspension culture in RPMI medium supplemented with penicillin/streptomycin and 5\% FBS.

Materials and reagents. The following reagents and antibodies were purchased: the p38 MAPK inhibitors, SB203580 (Sigma-Aldrich, St. Louis, MO, USA) and SB202190 (EMD Chemicals, Gibbstown, NJ, USA), the p44/42MAPK inhibitors, U0126 (Sigma-Aldrich) and PD98059 (EMD Chemicals), and the NADPH oxidase inhibitors, apocynin (Sigma-Aldrich) and diphenyliodonium (DPI) (SigmaAldrich). PD98059, SB203580, SB202190, DPI and apocynin were dissolved in $\mathrm{Me}_{2} \mathrm{SO}$. The final concentration of $\mathrm{Me}_{2} \mathrm{SO}$ was $<0.1 \%$ in these experiments. The terminal deoxynucleotidyl transferase-mediated dUTP nick endlabeling (TUNEL) cell apoptosis detection kit, JC-1 probe, the lactate dehydrogenase (LDH) activity kit and the caspase-3 activity assay kit, were provided by the Beyotime Institute of Biotechnology (Haimen, China). PCR primers were supplied by Sangon (Shanghai, China).

LOX-1 siRNA synthesis and transfection. Target sequences for human LOX-1 small interfering RNA (siRNA) were designed by GenBank, accession no. NM_002543.3, and siRNAs were synthesized by Invitrogen (Carlsbad, CA, USA). Different LOX-1 siRNAs were tested in the initial transfections and subsequent reverse transcription polymerase chain reaction (RT-PCR) and Western blot experiments. The oligonucleotides used to generate the LOX-1 siRNA chosen for use in this expriment were as follows: LOX-1-828 sense (named after the translation start site), 5'-CCCUUCAG GUACCUGUGCAUAUAUA-3' and LOX-1-828 antisense, 5'-UAUAUAUGCACAGGUACCUGAAGGG-3'. For the negative control, HRPTEpiC cultures were transfected with the control (non-targeting) siRNA duplex, designed by Invitrogen, with sequences not homologous to the genes in vertebrate transcriptome. The HRPTEpiCs were transfected in a culture vessel at $40-50 \%$ confluence, according to the manufacturer's instructions (Invitrogen). RNAi duplex and lipofectamine RNAiMAX were each diluted with Opti-
MEMI-reduced serum medium (Invitrogen). We then mixed the diluted RNAi duplex with the diluted lipofectamine RNAiMAX, and added this solution to the cells in a microplate to a final RNA concentration of $10 \mathrm{nM}$. After transfection for $6 \mathrm{~h}$, the supernatant was replaced with $2 \%$ FBS EpiCM and the cells were incubated for $24 \mathrm{~h}$. Finally, the cells were treated with $10^{-6} \mathrm{~mol} / \mathrm{l}$ Ang II for $24 \mathrm{~h}$ and then harvested.

RNA extraction and RT-PCR. Total RNA was extracted from the cells using TRIzol reagent (Invitrogen) according to the manufacturer's instructions. A reverse transcription kit (Gibco BR) was used to generate cDNA. First-stand cDNA was synthesized using oligonucleotide primers and M-MLV reverse transcriptase (Promega, Madison, WI, USA) before PCR amplification. Human LOX-1 primers (forward, 5'-TTA CTCTCCATGGTGGTGCC-3' and reverse, 5'-AGCTTCTT CTDCTTGTTGCC-3') and $ß$-actin (forward, 5'-CTCCAT CCTGGCCTCGCTGT-3' and reverse, 5'-GCTGTCACCT TCACCGTTCC-3'), were designed to amplify the respective regions. A 193-bp human LOX-1 cDNA and a 268-bp ß-actin cDNA fragment were amplified enzymatically in 30 and 28 cycles, respectively. Reaction mixtures were separated on $1.5 \%$ agarose gels containing ethidium bromide, and the bands were detected under UV light and analyzed with a Syngene bio-imaging system (Hercules, CA, USA).

Western blot analysis. The cells were lysed in RIPA lysis buffer (50 mM Tris, pH 7.4), $150 \mathrm{mM} \mathrm{NaCl,} 1 \%$ Triton X-100, $1 \%$ sodium deoxycholate, and $0.1 \%$ SDS). Protein concentrations were determined using a BCA protein assay kit (Beyotime). Samples were separated by $10 \%$ SDS-PAGE and then transferred to nitrocellulose membranes (Bio-Rad). After incubation in blocking solution (5\% non-fat milk; Sigma), the membranes were incubated with primary antibodies overnight at $4{ }^{\circ} \mathrm{C}$. The following antibodies were used: Rabbit polyclonal anti-LOX-1 antibody (1:5000; LifeSpan BioSciences, Seattle, WA, USA), mouse monoclonal antiICAM-1 antibody (MEM-111) (1:3000; Abcam, Cambridge, MA) and mouse monoclonal anti- $\beta$-actin antibody (1:5000; Sigma). Anti-rabbit and anti-mouse IgG secondary antibodies were conjugated with horseradish peroxidase (Beyotime, Jiangshu, China). Proteins were detected with the Amersham ECL chemiluminescence system (GE Healthcare, Piscataway, NJ, USA). The densities of protein bands relative to $B$-actin, were determined. The expression patterns of the $\mathrm{p} 38$, and p44/42 MAPK isoforms, the NADPH oxidase subunits (p22phox, p47phox and Nox-4) and cytochrome c (1:1000) were also assessed by Western blot analysis.

Isolation of cytosolic fraction for cytochrome c analysis. After the cells were treated with Ang II in the presence or absence of LOX-1 siRNA, they were collected and lysed with lysis buffer (20 mM HEPES/NaOH, pH 7.5, $250 \mathrm{mM}$ sucrose, $10 \mathrm{mM}$ $\mathrm{KCl}, 2 \mathrm{mM} \mathrm{MgCl}$, $1 \mathrm{mM}$ EDTA, $1 \mathrm{mM}$ DTT and protease inhibitor cocktail) for $20 \mathrm{~min}$ on ice. The samples were then homogenized by 10 passages through a 2-gauge needle and the homogenate was then centrifuged at $12,000 \mathrm{rpm}$ for $20 \mathrm{~min}$ at $4^{\circ} \mathrm{C}$. A volume of cell lysates containing $30 \mu \mathrm{g}$ of protein was analyzed by Western blot analysis. 


\section{A}
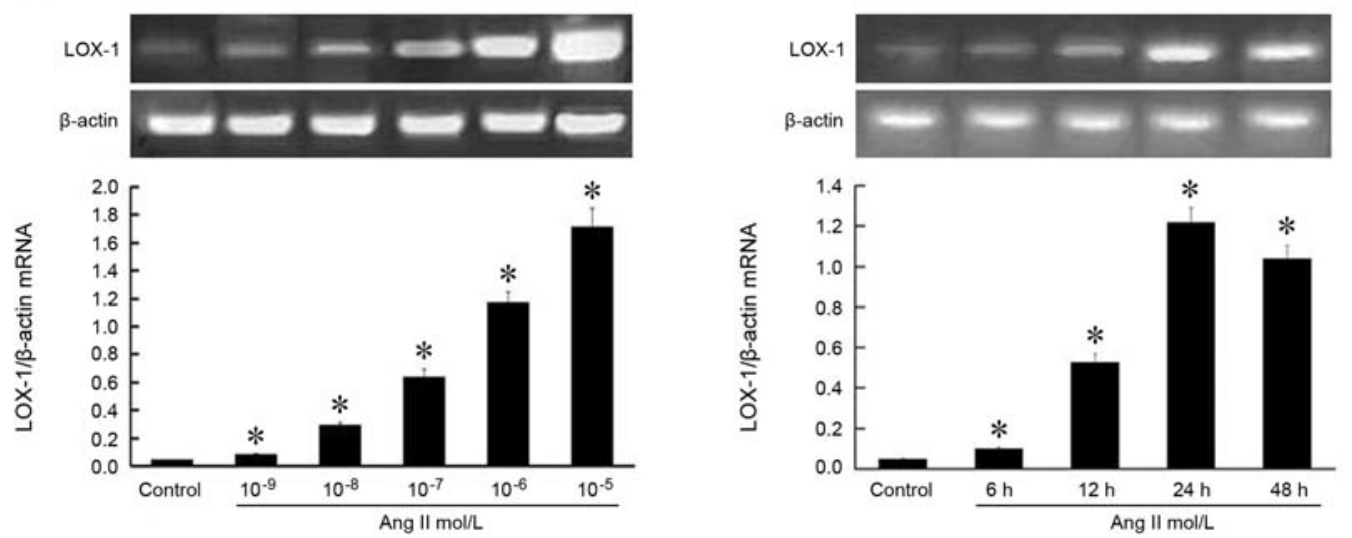

B
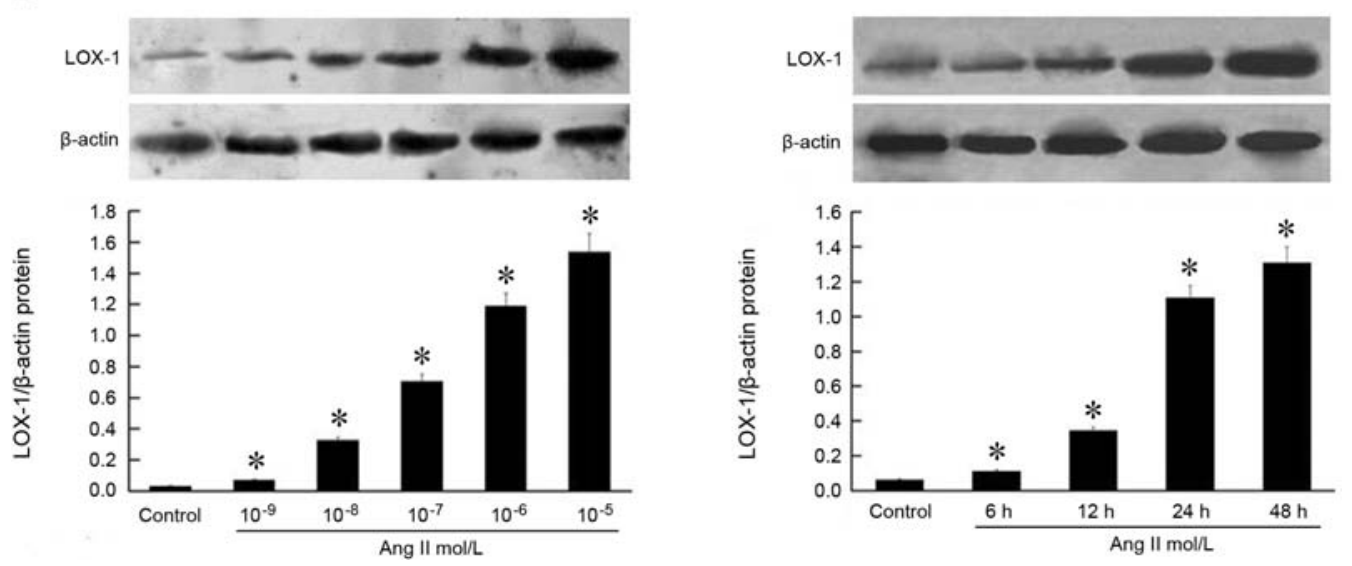

Figure 1. Ang II-induced LOX-1 expression in HRPTEpiCs. Cultured HRPTEpiCs were incubated for 6-48 h with $10^{-6} \mathrm{~mol} / 1 \mathrm{Ang}$ II or $24 \mathrm{~h}$ with $10^{-9}-10^{-5} \mathrm{~mol} / 1$ Ang II. At the end of the incubation period, the cells were lysed and LOX-1 mRNA and protein levels were analyzed by RT-PCR (A) and Western blotting (B). LOX-1 mRNA and protein levels were normalized to the levels of $\beta$-actin. Data represent the means \pm SEM of 3 different experiments. ${ }^{*} \mathrm{P}<0.01$ vs. control. The top panels are representative of 3 separate experiments. The bottom panels show a summary of the data (means \pm SEM) from these 3 experiments.

Adhesion assay. HRPTEpiCs were cultured in 6-well flatbottomed plates for 1 day. The cells were then pre-transfected with LOX-1 siRNA as previously described and U937 cells [pre-labeled with $5 \mathrm{mg} / 1$ BCECF-AM (Sigma-Aldrich) for $30 \mathrm{~min}$ in RPMI at a density of $4 \times 10^{6}$ cell $/ \mathrm{ml}$ ] were then added. The cells were allowed to adhere at $37^{\circ} \mathrm{C}$ for $1 \mathrm{~h}$ in a $5 \% \mathrm{CO}_{2}$ incubator. The non-adherent cells were removed by gentle aspiration. The plates were washed 3 times with EpiCM before lysis with $0.1 \%$ Triton X-100. The number of adherent cells was estimated by microscopic examination. Fluorescence intensity was measured at 485-nm excitation and 538-nm emission using a Labsystems fluorescence microplate reader.

Determination of cytotoxicity. At the end of stimulation, mitochondrial dehydrogenase activity, which can be used as an index of cell viability, was assessed using the previously described 3-(4,5-dimethylthiazol-2-yl)-2,5-diphenyltetrazolium (MTT) assay (11). Plasma membrane integrity was assessed by measuring LDH release using an LDH diagnostic kit according to the manufacturer's instructions.

Measurement of reactive oxygen species (ROS) in HK-2 cells. Intracellular ROS generation was measured using 5(6)-chloromethyl-2',7'-dichlorodihydrofluorescein diacetate (CM-H $\mathrm{H}_{2} \mathrm{DCFDA}$; Invitrogen), a cell-permeable ROS indicator, at a concentration of $10 \mu \mathrm{M}$. The cells were pre-transfected with LOX siRNA as indicated above, then Ang II was added for $2 \mathrm{~h}$. Finally, the cells were rinsed with PBS and CM$\mathrm{H}_{2}$ DCFDA was added. Fluorescence intensity was measured using a Labsystems fluorescence microplate reader. Results are expressed as ratios to the control conditions.

Measurement of mitochondrial membrane potential. A JC-1 probe was employed to measure mitochondrial depolarization in the HRPTEpiCs. JC-1 exists either as a green fluorescent monomer at depolarized membrane potentials or as a red fluorescent J-aggregate at hyperpolarized membrane potentials. JC-1 exhibits potential-dependent accumulation in mitochondria, which is indicated by a fluorescence emission shift from 529 to $590 \mathrm{~nm}$. After the HRPTEpiCs were treated with $10^{-6} \mathrm{~mol} / \mathrm{l}$ Ang II for $24 \mathrm{~h}$ in the presence or absence of LOX-1 siRNA, the cells were rinsed with medium, and JC-1 was loaded. After $20 \mathrm{~min}$ of incubation at $37^{\circ} \mathrm{C}$, the cells were examined under an Olympus IX70 fluorescence microscope (Japan). Mitochondrial depolarization was indicated by an increase in the green/red fluorescence intensity ratio.

TUNEL. The TUNEL method was performed to label the 3'ends of fragmented DNA in apoptotic HRPTEpiCs. After the HRPTEpiCs were treated as indicated above, the cells were 
rinsed with PBS, permeabilized with $0.1 \%$ Triton $\mathrm{X}-100$, and finally incubated with TUNEL labeling mix for $60 \mathrm{~min}$ at $37^{\circ} \mathrm{C}$. FITC-labeled TUNEL-positive cells were imaged by fluorescence microscopy with a 488-nm excitation and 530-nm emission filter.

Caspase-3 activity. After lysis by repeated freeze-thaw, the cells were incubated on ice for 15 min and then centrifuged at $15,000 \mathrm{x} g$ for $20 \mathrm{~min}$. Protein concentrations were determined by the Bradford assay. Caspase- 3 activity was measured by the cleavage of the chromogenic caspase substrate, acetylAsp-Glu-Val-Asp p-nitroanilide (Ac-DEVD-pNA). Total protein $(\sim 40 \mu \mathrm{g})$ was added to the reaction buffer containing Ac-DEVD-pNA $(2 \mathrm{mM})$. The resulting solution was incubated for $2 \mathrm{~h}$ at $37^{\circ} \mathrm{C}$, and then the absorbance of yellow pNA cleaved from its precursor was measured using a spectrometer at $405 \mathrm{~nm}$. Caspase-3 activity, normalized to the total protein concentrations of the cell lysates, was then expressed as a fold change compared to the baseline caspase activity of the control cells cultured in EPiCM with 2\% FBS.

Statistical analysis. Data were analyzed by one-way ANOVA (analysis of variance), and multiple comparisons between the treatment groups were made using the Dunnett or Bonferroni tests. The results were expressed as the means \pm SEM. Values of $\mathrm{P}<0.05$ were considered statistically significant.

\section{Results}

Induction of LOX-1 gene and protein expression by Ang II in HRPTEpiCs. The luminal expression of LOX-1 in renal epithelial cells is a novel finding. Accordingly, evidence of the direct effects of oxLDL on the tubular epithelium was sought in cultured NRK52E cells, which are derived from normal rat proximal tubules (2). The incubation of HRPTEpiCs for $24 \mathrm{~h}$ with increasing Ang II concentrations $\left(10^{-9}-10^{-5} \mathrm{~mol} / \mathrm{l}\right)$ enhanced LOX-1 mRNA and protein expression in these cells in a dose-dependent manner. Maximal effects were observed with a concentration of $10^{-5} \mathrm{~mol} / \mathrm{l}$ Ang II. Treatment of the HRPTEpiCs for 6-48 h with $10^{-6} \mathrm{~mol} / \mathrm{l}$ Ang II, caused LOX-1 mRNA and protein levels to markedly increase in a timedependent manner, with maximal effects on LOX-1 protein expression at $48 \mathrm{~h}$. The LOX-1 mRNA levels, normalized to the $B$-actin mRNA levels, are shown in Fig. 1A. The LOX-1 protein levels normalized to the $B$-actin levels are shown in Fig. 1B.

Role of LOX-1 in Ang II-induced ICAM-1 expression and adherence of U937 cells to HRPTEpiCs. In order to determine whether the Ang II-induced ICAM-1 expression is mediated by LOX-1, we conducted a series of silencing experiments with LOX-1 siRNA. As previously described, the Ang IItreated cultured tubular epithelial cells showed the same upregulation of LOX-1 and ICAM-1 protein after 24-h exposure as did the untreated cells. In contrast, the LOX-1 up-regulation was almost abolished when the cells were transfected with 10 nM LOX-1 siRNA before exposure to Ang II. The expression analysis of ICAM-1 gave a similar result (Fig. 2A). Specifically, the LOX-1 knockdown was sufficient to prevent ICAM-1 expression after the treatment with Ang II. In parallel
A
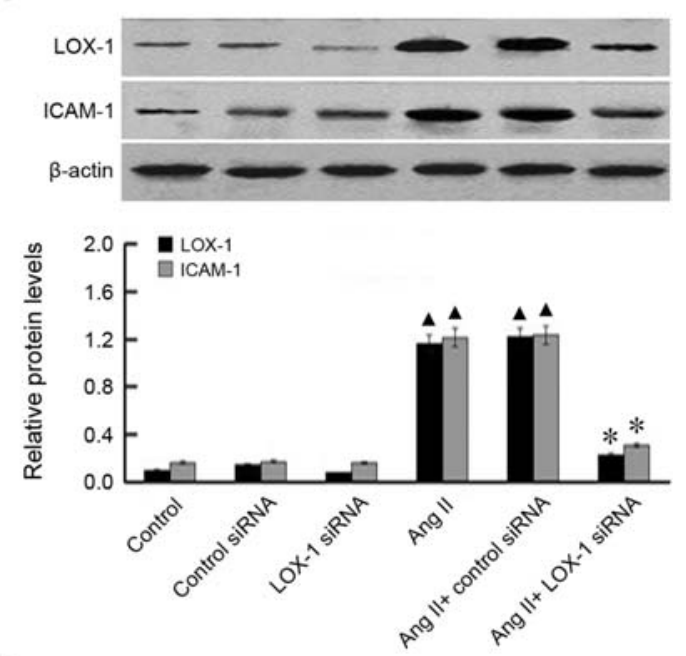

B

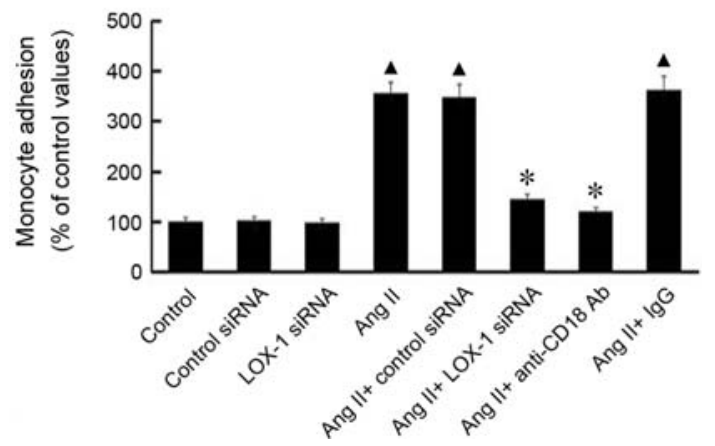

Figure 2. LOX-1 and ICAM-1 protein expression (A), and human monocyte adhesion to HRPTEpiCs (B). (A) Western blot analysis of LOX-1 and ICAM-1 protein expression in HRPTEpiCs exposed for $24 \mathrm{~h}$ to $10^{-6} \mathrm{~mol} / \mathrm{l}$ Ang II in the presence of LOX-1 siRNA. Protein bands were quantified by densitometry. (B) Confluent HRPTEpiCs were exposed for $24 \mathrm{~h}$ to $10^{-6} \mathrm{~mol} / \mathrm{l}$ Ang II in the presence of LOX-1 siRNA or anti-CD18 antibodies. At the end of this incubation period, the cells were washed and monocytes were added to the HRPTEpiCs in order to measure monocyte adhesion. Data are expressed as the percentage of adherent monocytes and represent the means \pm SEM of 5 different experiments. ${ }^{\wedge} \mathrm{P}<0.01$ vs. control; ${ }^{*} \mathrm{P}<0.01$ vs. Ang II group or Ang II + control siRNA group.

experiments, we sought to determine whether the Ang IIinduced monocyte adhesion to the HRPTEpiCs is mediated by LOX-1. Confluent monolayers of HRPTEpiCs were pretreated with LOX-1 siRNA and then stimulated with Ang II for $24 \mathrm{~h}$, followed by incubation with U937 cells for $2 \mathrm{~h}$ at $37^{\circ} \mathrm{C}$. As shown in Fig. 2B, Ang II stimulated an increase in the adherence of U937 cells to HRPTEpiCs $(356 \pm 23 \%$, $\mathrm{P}<0.01)$. However, the effect was significantly inhibited after treatment with LOX-1 siRNA $(144 \pm 10 \%, \mathrm{P}<0.01)$. In order to test the involvement of ICAM-1 in the Ang II-induced monocyte adhesion to the HRPTEpiCs, we incubated the monocytes with Ang II-treated HRPTEpiCs in the presence of anti-CD18 antibody $(10 \mathrm{mg} / \mathrm{l})$. The addition of the antibody lead to a significant decrease in ICAM-1-dependent monocyte binding. Non-specific IgG had no effect. These data suggest that the suppression of endogenous LOX-1 inhibits Ang IIinduced ICAM-1 expression and monocyte adhesion.

LOX-1 mediated Ang II-induced cytotoxicity in HRPTEpiCs. In order to examine the effects of Ang II on the morpho- 


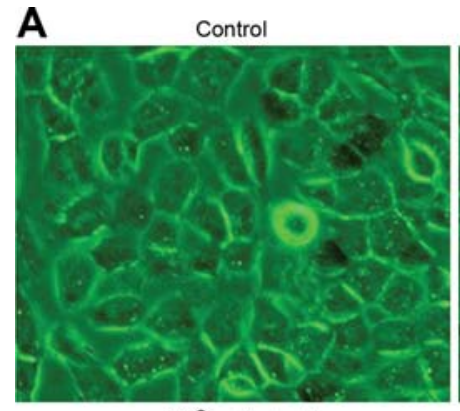

$10^{-6} \mathrm{~mol} / \mathrm{L}$ Ang $\|$

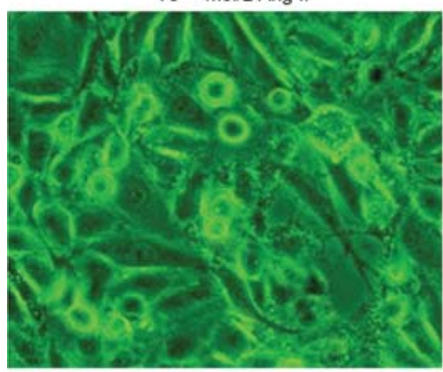

B

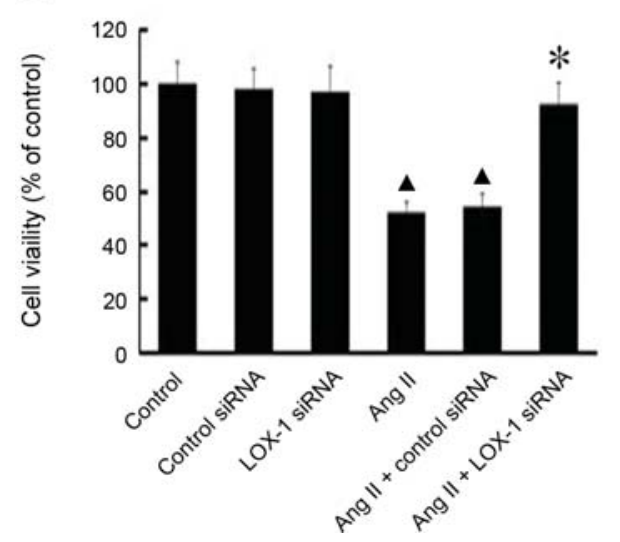

Control siRNA

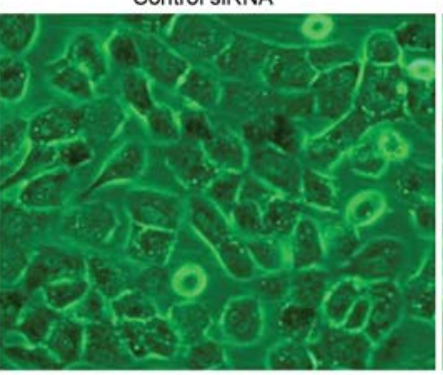

Ang II + control siRNA

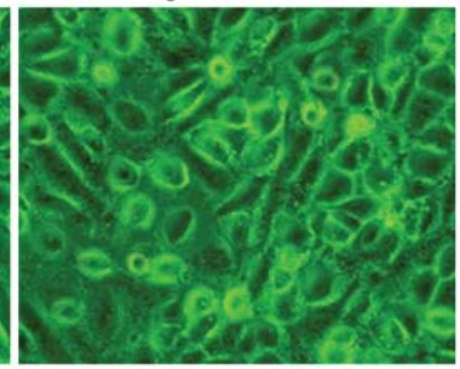

LOX-1 SIRNA

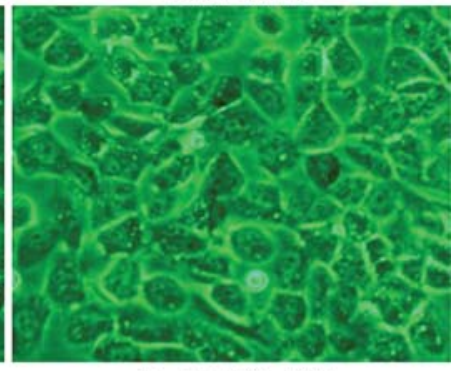

Ang $\|+$ LOX-1 siRNA

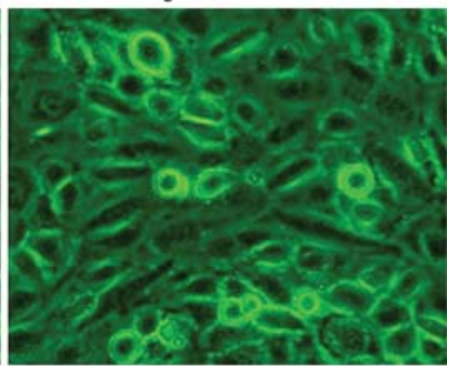

\section{C}

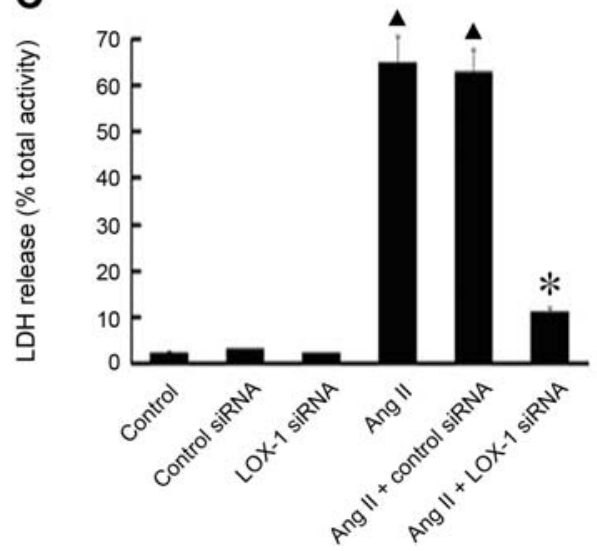

Figure 3. Effect of LOX-1 siRNA treatment on Ang II-induced cell death in HRPTEpiCs. HRPTEpiCs were treated as described in Fig. 2A. Photomicrographs are from phase-contrast microscopy (A). Viability was determined via MTT assay (B) and lactate dehydrogenase (LDH) release (C). Values are the means \pm SEM of 3 separate experiments. ${ }^{\wedge} \mathrm{P}<0.01$ vs. control; ${ }^{*} \mathrm{P}<0.01$ vs. Ang II group or Ang II + control siRNA group. Magnification, $\mathrm{x} 400$, phase-contrast microscopy.

logical features of HRPTEpiCs, phase-contrast microscopy (Olympus IX70, Japan) was performed (Fig. 3A). The number of shrunken cells and cells with blebbing membranes significantly increased after 24-h exposure to Ang II. This cytotoxic effect was prevented by treating the cells with LOX-1 siRNA. The viability of the cells incubated with Ang II in the presence or absence of LOX siRNA was assessed by MTT assay (Fig. 3B), and membrane permeability was assayed by LDH release (Fig. 3C). The treatment of confluent HRPTEpiCs with Ang II for $24 \mathrm{~h}$ resulted in a significant decrease in cellular viability and an increase in membrane permeability compared to the control cells and those preincubated with LOX-1 siRNA. These results suggest that LOX-1 knockdown is a potent inhibitor of Ang II-induced cytotoxicity in cultured HRPTEpiCs.

Role of LOX-1 in Ang II-induced apoptosis in HRPTEpiCs. As the inhibition of mitochondrial disruption accounts for the anti-apoptotic effect, we tested the effects of LOX-1 siRNA on the AngII-induced mitochondrial permeability. Green fluorescence indicated the cells with a low $\Delta \Psi \mathrm{m}$, while red fluorescence indicated the cells with a stable $\Delta \Psi \mathrm{m}$. When the HRPTEpiCs were exposed to Ang II, $\Delta \Psi \mathrm{m}$ was depolarized compared to the control, as indicated by an increase in green fluorescence (Fig. 4). Pre-treatment with LOX-1 siRNA reduced the effect on $\Delta \Psi \mathrm{m}$, as indicated by the repression of green fluorescence and the restoration of red fluorescence (Fig. 4). The effect of Ang II was dependent on LOX-1 function, as LOX-1 siRNA treatment resulted in a significant inhibition of this Ang II-induced apoptotic index.

In order to further ascertain whether the Ang II-induced apoptosis is mediated by LOX-1, the Ang II-treated cells were analyzed biochemically via TUNEL apoptosis assay and evaluated by fluorescence microscopy. As shown in Fig. 5, the cells incubated with Ang II for $24 \mathrm{~h}$ showed an increase in green fluorescence and condensed nuclei, indicating apoptosis. These features were barely observed in the untreated cells or those pre-treated with LOX-1 siRNA. These results 


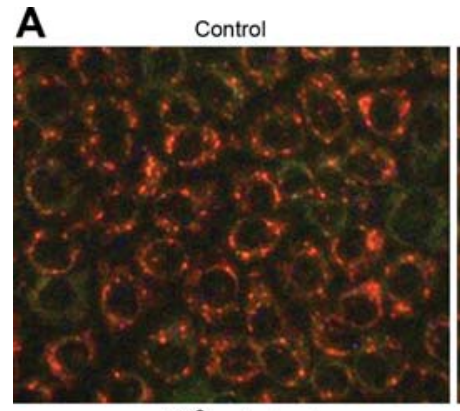

$10^{-6}$ moll. Ang II
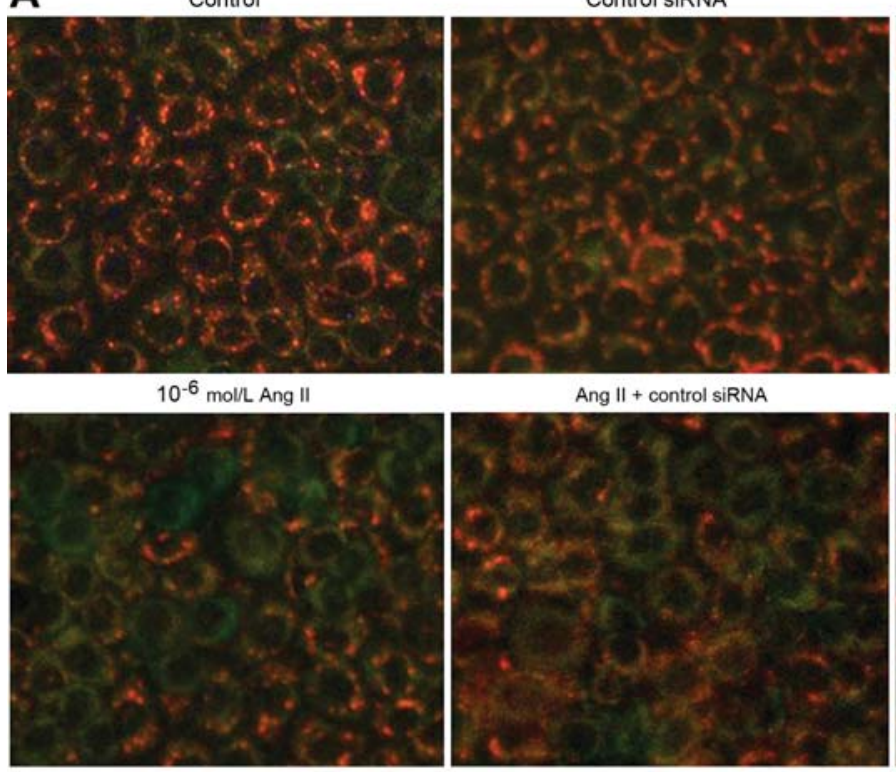

Ang $\|+$ control siRNA

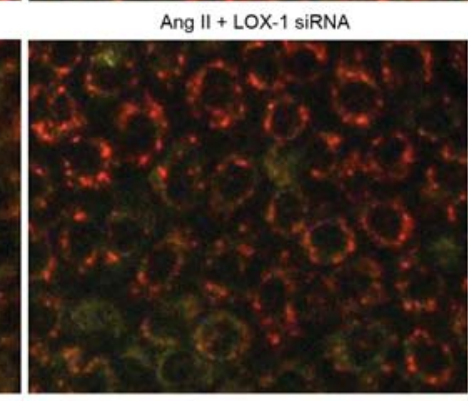

LOX-1 SIRNA

Ang II + LOX-1 siRNA
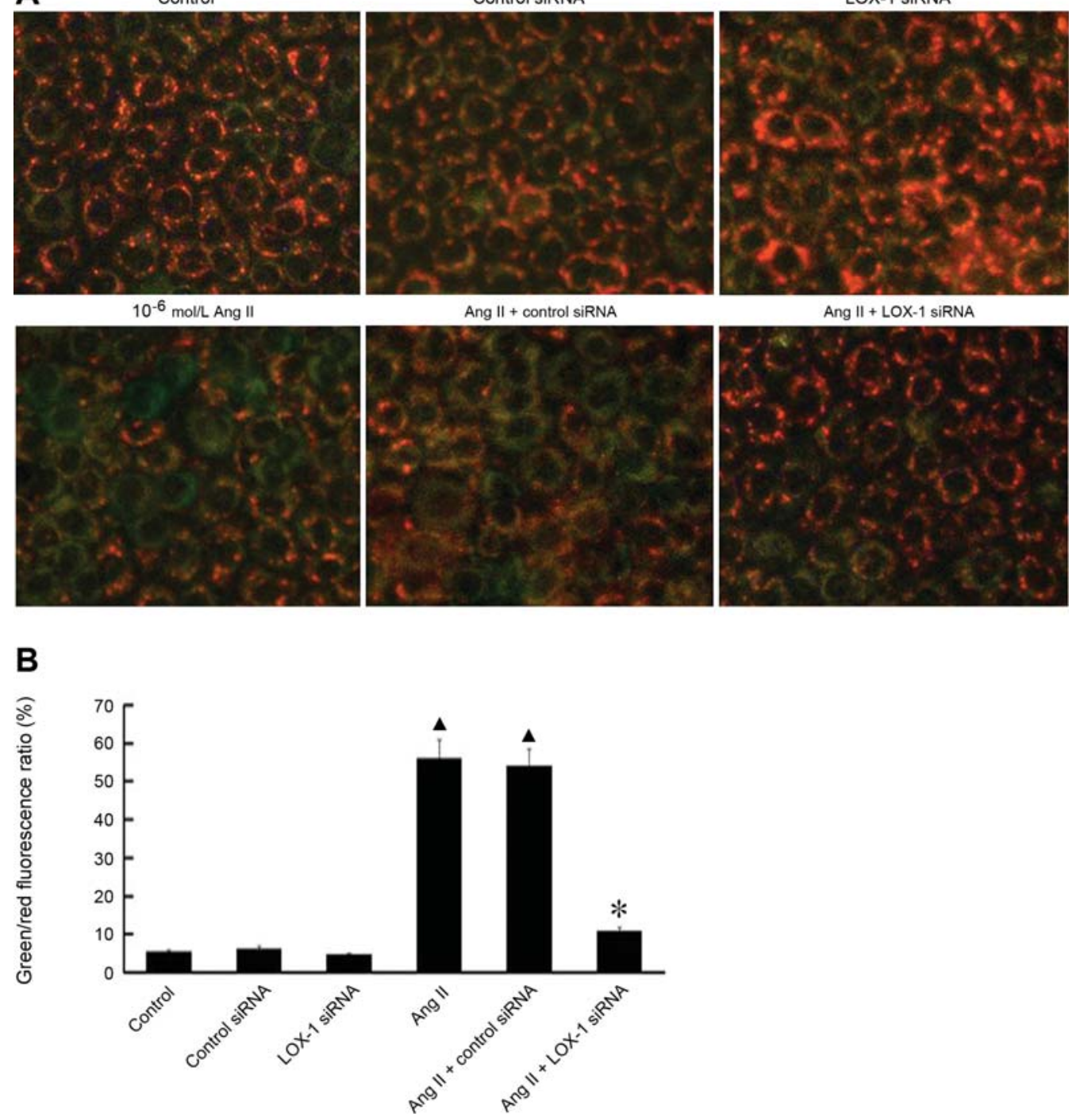

Figure 4. Effects of LOX-1 siRNA on mitochondrial potential reduction in Ang II-induced HRPTEpiCs. HRPTEpiCs were treated as described in Fig. 2A. (A) The cells were stained with JC-1 probe and imaged by fluorescence microscopy (magnification, $\mathrm{x} 400$ ). Average red and green fluorescence intensities are expressed as the ratio of green to red fluorescence. An increase in the bar indicates a shift in the fluorescence ratio, which signals an increase in mitochondrial depolarization. (B) Quantitative analysis of the shift of mitochondrial orange-red to green fluorescence among the groups. All values are the means \pm SEM from 10 independent micrographs from each group.

demonstrate that LOX-1 siRNA inhibits apoptosis in Ang IItreated cells, supporting the hypothesis that Ang II-induced apoptosis is mediated by LOX-1 in these cells.

In order to provide additional support for this hypothesis, we observed the effects of Ang II on the activation of the mitochondrial pathway of apoptosis. Bcl-2 family proteins are upstream regulators of mitochondrial membrane potential. Immunoblotting studies demonstrated that Ang II downregulated the anti-apoptotic (Bcl-2) and stimulated the proapoptotic (Bax) proteins, whereas the pre-treatment with LOX-1 siRNA effectively repressed these Ang II-induced pro-apoptotic events. Based on quantitative analysis, Ang II significantly decreased the Bcl-2-to-Bax ratio, but the addition of LOX-1 siRNA preserved this anti-apoptotic index (Fig. 6A-a).

It is known that the disruption of mitochondrial membrane function results in the specific release of the mitochondrial enzyme, cytochrome c, into the cytosol. Therefore, mito- chondria were separated from the cytosolic fraction and the amount of cytochrome c released into the cytosolic fraction was detected by Western blotting. As shown in Fig. 6A-b, the amount of cytochrome $\mathrm{c}$ released was much greater in the HRPTEpiCs that had been incubated with Ang II for $24 \mathrm{~h}$ than in the control cells. Pre-treatment with LOX-1 siRNA resulted in a decrease in Ang II-mediated cytochrome c release compared to the control Ang II-treated cells. However, treatment with non-targeting siRNA had no effect on Ang IIdependent cytochrome c levels. These results indicate that that the LOX-1 knockdown prevents the Ang II-induced release of cytochrome $\mathrm{c}$.

Caspase-3 is one of the downstream effectors of the caspase family, and is involved in both the mitochondrial apoptotic and the death receptor pathway. Caspase- 3 is synthesized as a precursor protein, pro-caspase-3, which undergoes cleavage and subsequent activation in response to apoptotic stimuli by initiator caspases (including caspase-2, -8, 

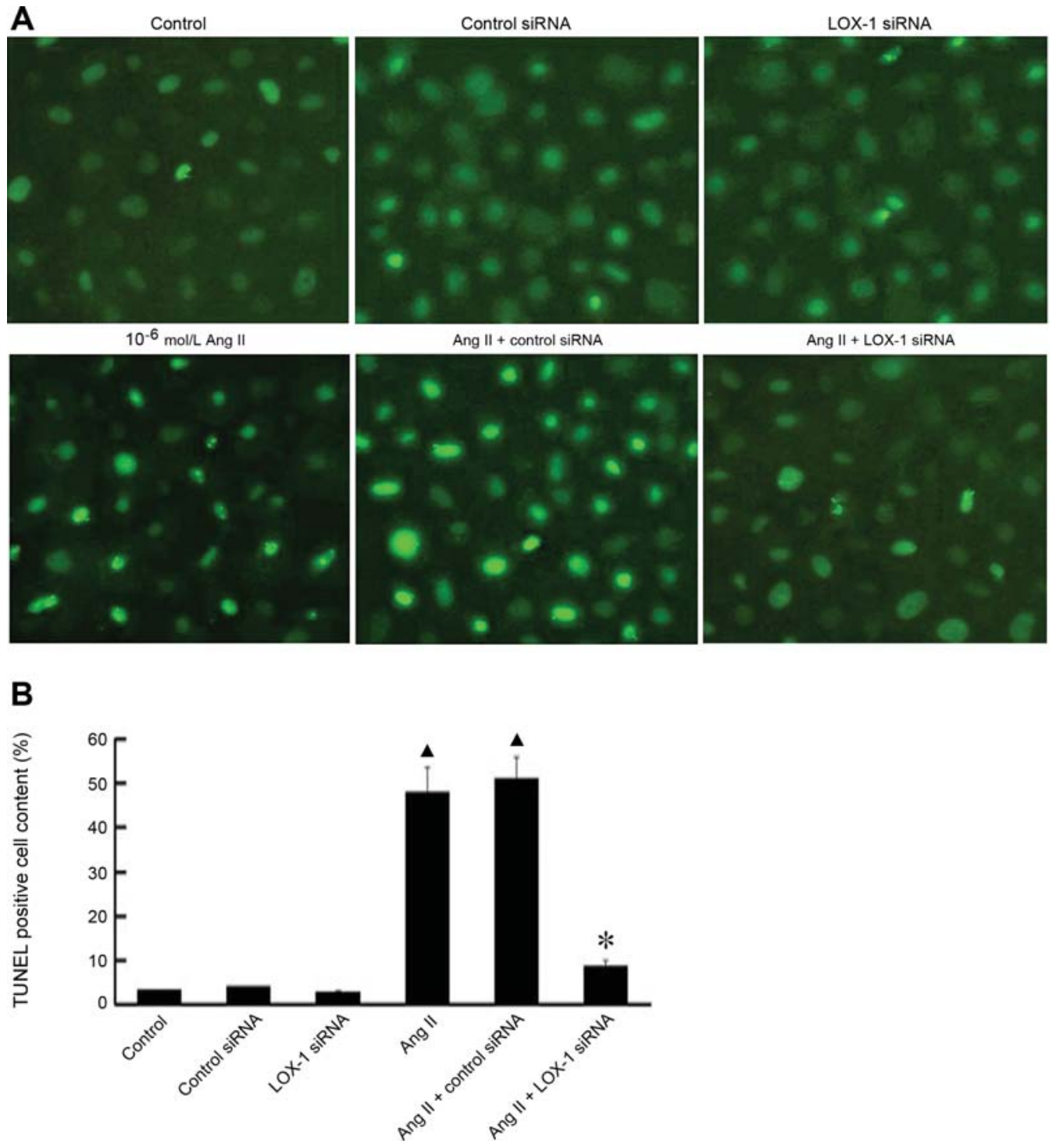

Figure 5. TUNEL-positive cells. HRPTEpiCs were treated as described in Fig. 2A before TUNEL staining and fluorescence imaging. (A) Fluorescence microscopy shows TUNEL staining in Ang II-induced HRPTEpiCs with or without LOX-1 siRNA transfection (magnification, x200). (B) The quantity of TUNEL-positive cells was calculated as the ratio of TUNEL-positive cells to the total number of HRPTEpiCs. All values are the means \pm SEM from 10 independent micrographs from each group. ${ }^{\mathbf{P}}<0.01$ vs. control; ${ }^{*} \mathrm{P}<0.01 \mathrm{vs}$. Ang II group or Ang II + control siRNA group.

$-9,-10,-11$ and -12) (12). In the present study, we found that the treatment of cells with $10^{-6} \mathrm{~mol} / \mathrm{l}$ Ang II promoted higher levels of cleavage and activation of pro-caspase-3 compared to the control cells in normal culture medium. Additionally, LOX-1 siRNA inhibited pro-caspase-3 cleavage, although it did not completely restore the amount of pro-caspase- 3 to the control level (Fig. 6B).

Interaction between Ang II, LOX-1 and NADPH oxidase. It is now well accepted that endocrine and paracrine Ang II activates intracellular signaling pathways and induces diverse biological effects via the release of ROS (13). NADPH oxidase activation is a major source of ROS in renal cells (14). Therefore, we measured intracellular ROS generation and NADPH oxidase expression in Ang II-induced HRPTEpiCs. As predicted, $10^{-6} \mathrm{~mol} / 1$ Ang II enhanced the expression of NADPH oxidase (the p22phox, p47phox and Nox-4 subunits) and increased dihydrofluorescein (DCF) fluorescence, which reflects intracellular ROS generation.
Previous studies have shown that oxidative stress upregulates the expression and activation of LOX-1, and that the activation of LOX-1 itself can stimulate the formation of ROS (15). In the present study, the treatment of HRPTEpiCs with apocynin and DPI, 2 NADPH oxidase inhibitors, attenuated the expression of LOX-1 (Fig. 8A). Here, we also found that LOX-1 siRNA treatment significantly reduced the Ang II-induced expression of NADPH oxidase (all 3 subunits) (Fig. 7A) and intracellular ROS generation (Fig. 7B). Non-targeting siRNA had no effect on the Ang II-induced expression of NADPH oxidase and ROS generation. These observations suggest that Ang II, as a mediator of oxidative stress, induces LOX-1 expression, which results in the activation of NADPH oxidase and ROS generation, which in turn up-regulates LOX-1 expression.

Apocynin and DPI also inhibited the expression of ICAM-1 (Fig. 8A) and down-regulated caspase-3 activity (Fig. 8B), a key factor in the execution of mitochondrial apoptosis. In addition, LOX-1 knockdown reduced ICAM-1 
A

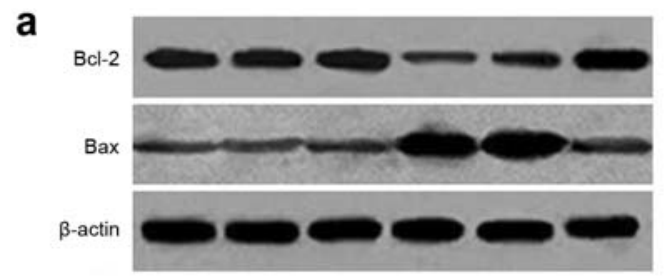

b
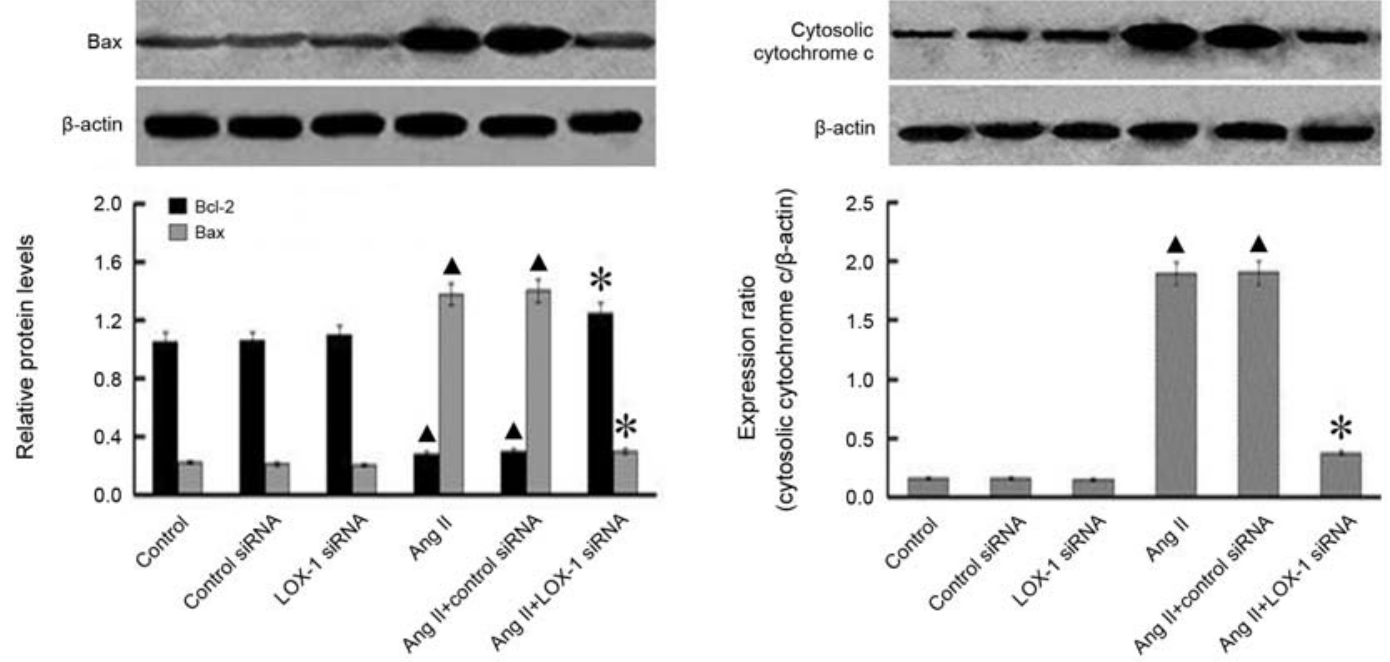

B

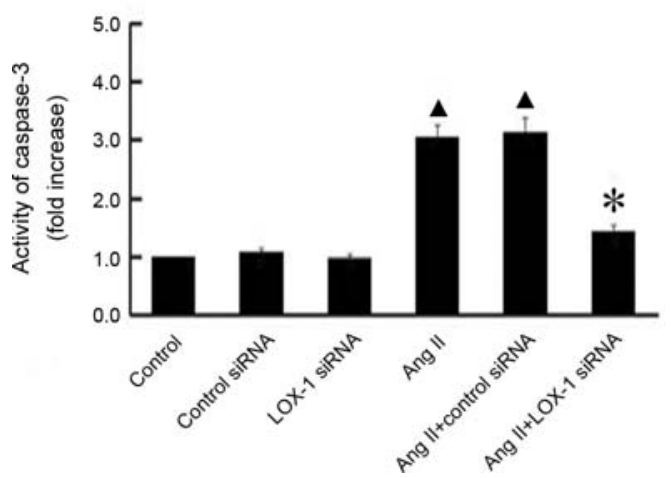

Figure 6. Ang II induces apoptosis in HRPTEpiCs through LOX-1 and exerts opposite effects on pro-survival and pro-apoptotic targets. (A) Bcl-2 and Bax expression (a) and mitochondrial cytochrome c release (b) were evaluated by Western blotting and protein levels were normalized to B-actin. (B) Caspase-3 activation in HRPTEpiCs was measured using the chromogenic substrate Ac-DEVD-pNA. The bar represents the fold increase in pNA absorbance produced by the cleavage of its precursor in the HRPTEpiCs. $\triangle \mathrm{P}<0.01$ vs. control; ${ }^{*} \mathrm{P}<0.01$ vs. Ang II group or Ang II + control siRNA group. (n=3).

expression, monocyte adhesion and apoptosis. These results demonstrate that Ang II up-regulates the expression of LOX-1 and NADPH oxidase, and that LOX-1 promotes further ROS generation and oxidative functional damage, inducing ICAM-1 expression, cytotoxicity and apoptosis.

Signaling of Ang II-LOX-1-mediated oxidative functional damage. Certain members of the MAPK family of proteins are activated by the stimulation of LOX-1. These proteins are involved in changing the normally quiescent epithelial phenotype of tubular cells into a pro-inflammatory one, which is characterized by greater epithelial permeability, increased ICAM-1 expression, enhanced leukocyte adherence and apoptosis (2). Hence, it was logical to test the potential of Ang II in activating and phosphorylating the MAPK proteins, p38 and p44/42, in kidney cells. We measured the expression of the redox-sensitive p38 and p44/42 MAPKs and found that Ang II markedly increased the phosphorylation of both the p38 and p44/42 MAPKs at 20 min $(\mathrm{P}<0.01)$. This phosphorylation was inhibited by the addition of LOX-1 siRNA.
Non-targeting siRNA had no effect (Fig. 9). In order to determine whether the anti-oxidative pathway plays a role in Ang II-mediated signaling cascades, apocynin and DPI were used to pre-treat renal tubular epithelial cells before MAPK protein level determination. Apocynin and DPI also significantly reduced the phospho-p38 and -p44/42 protein levels in the Ang II-treated cells (Fig. 9).

In order to study the intracellular signaling mechanism of the LOX-1-Ang II interaction, the cells were treated with a variety of inhibitors. As shown in Fig. 8, the treatment of cells with NADPH oxidase inhibitors (apocynin and DPI), p44/42 MAPK inhibitors (U0126 and PD98059), or p38 MAPK inhibitors (SB203580 and SB202190) reduced the expression of ICAM-1 (Fig. 8A) and suppressed caspase-3 activity (Fig. 8B). Importantly, the Ang II-mediated upregulation of LOX-1 was inhibited by NADPH oxidase inhibitors, but not by MAPK inhibitors (Fig. 8A), indicating that NADPH oxidase activation plays a significant role in Ang II-mediated LOX-1 expression, and that NADPH oxidase activation is upstream of MAPKs. 
A
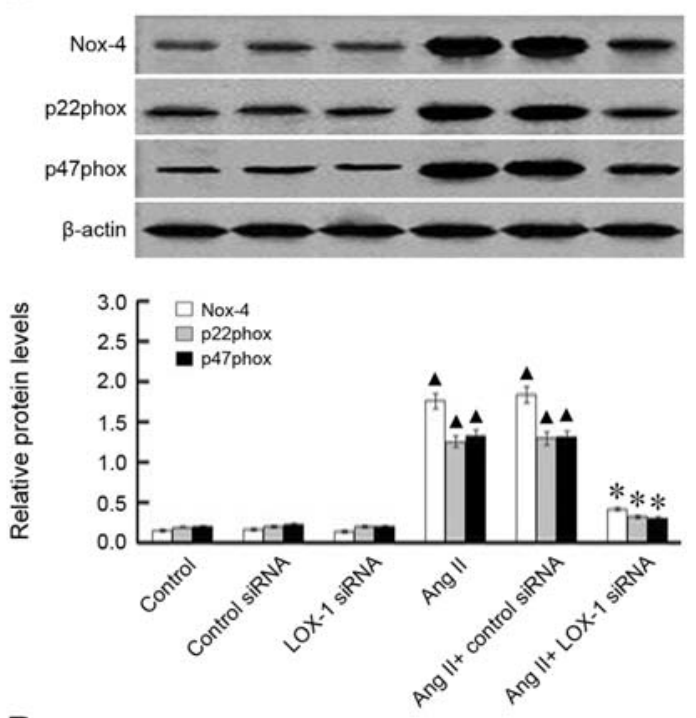

B

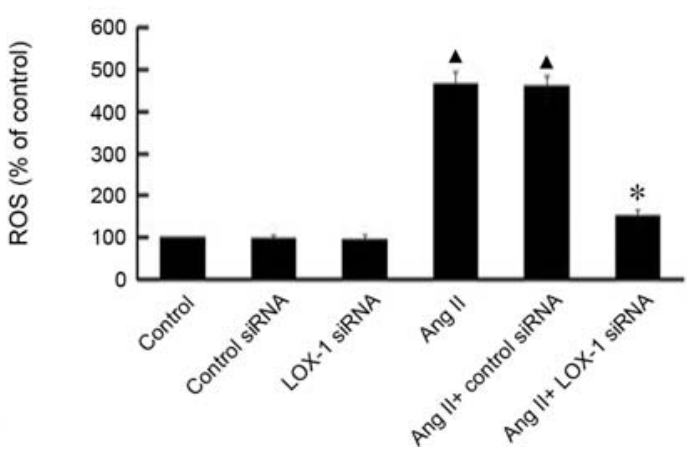

Figure 7. Effect of LOX-1 siRNA transfection on NADPH oxidase expression and ROS generation in HRPTEpiCs induced by $10^{-6} \mathrm{~mol} / 1 \mathrm{Ang}$ II. Ang II enhanced the expression of the NADPH oxidase subunits p22phox, p47phox and NOX4phox (A) and increased ROS generation as measured by 2',7'-DCF fluorescence (B). The treatment of cells with LOX-1 siRNA reduced NADPH oxidase (p22phox, p47phox and gp91phox) expression and DCF fluorescence. Non-targeting siRNA had no effect on NADPH oxidase expression or DCF fluorescence. $\triangle \mathrm{P}<0.01$ vs. control; ${ }^{*} \mathrm{P}<0.01$ vs. Ang II group or Ang II + control siRNA group.

A
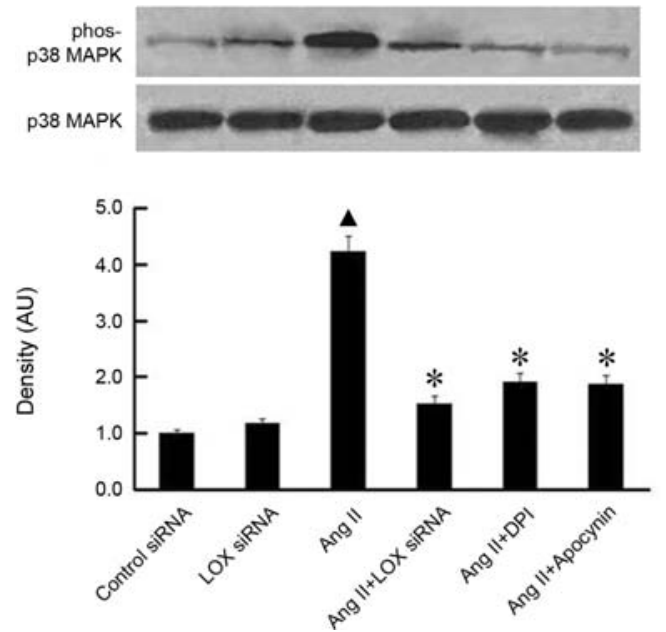

A

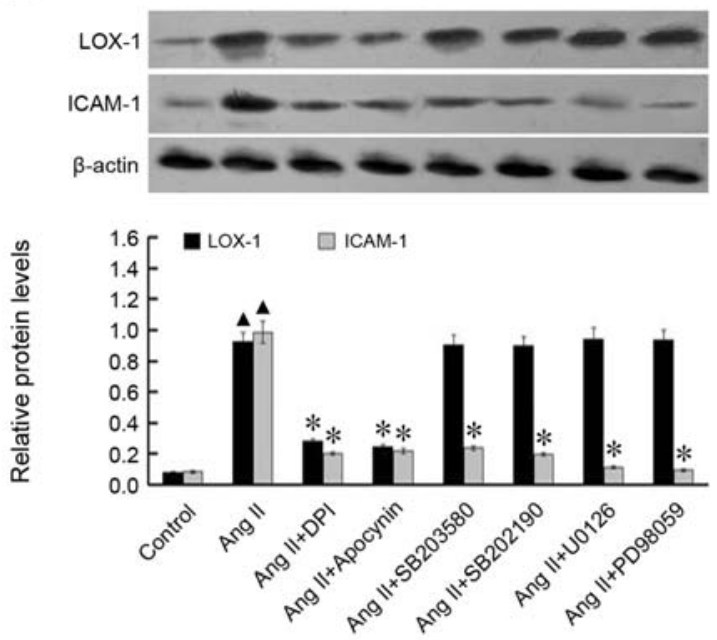

B

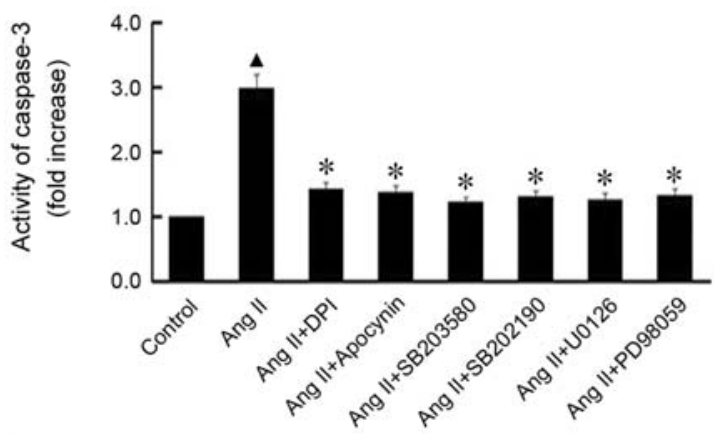

Figure 8. Effects of NADPH oxidase and MAPKs inhibitors on LOX-1 and ICAM-1 expression (A) and caspase-3 activation (B). The expressions of LOX-1 and ICAM-1 were increased in the HRPTEpiCs after the treatment with Ang II. The NADPH oxidase inhibitors, apocynin and DPI, inhibited LOX-1 and ICAM-1 expression and caspase- 3 activity. The p38 and p44/42 MAPK inhibitors supressed ICAM- 1 expression and caspase- 3 activity but had no effect on LOX-1 expression. $\triangle \mathrm{P}<0.01$ vs. control; ${ }^{*} \mathrm{P}<0.01$ vs. Ang II group.

\section{B}
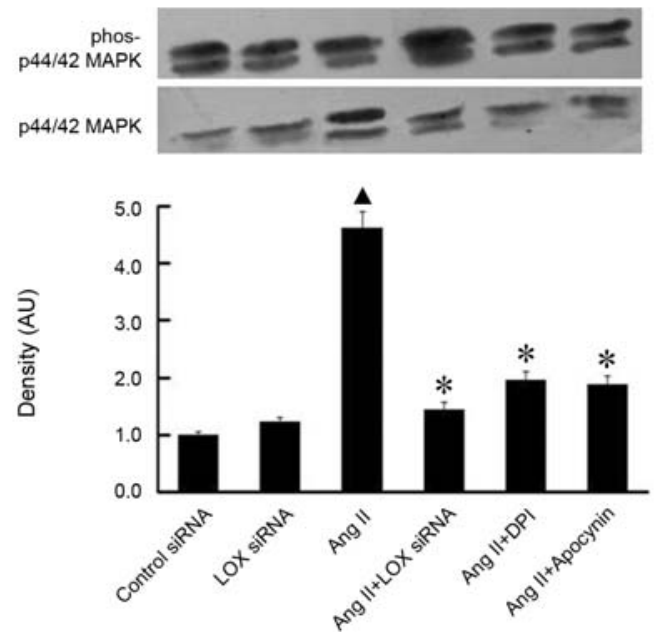

Figure 9. Western blot analysis of MAPK expression. Protein levels of p38 (A) or p44/42 MAPKs (B) were not altered by the control siRNA or LOX siRNA alone. However, Ang II markedly increased the phosphorylation of both p38 and p44/42 MAPKs, which was inhibited by LOX-1 siRNA, apocynin and DPI. $\Delta \mathrm{P}<0.01$ vs. control siRNA or LOX siRNA; ${ }^{*} \mathrm{P}<0.01$ vs. Ang II group. 


\section{Discussion}

Many studies have demonstrated the inappropriate activation of the intrarenal renin-angiotensin system and the overexpression of LOX-1 in the progression of renal diseases $(1,16)$. It has been shown that the local AT1 receptor (AT1r) activation by Ang II causes the up-regulation/activation of $\mathrm{NAD}(\mathrm{P}) \mathrm{H}$ oxidase and oxidative stress in kidney and cardiovascular tissues (17). In addition, Ang II has been found to promote the activation of $\mathrm{NF}-\kappa \mathrm{B}$ and the production of proinflammatory cytokines, chemokines, growth factors and adhesion molecules, which cause inflammation and fibrosis $(18,19)$. In the present study, we demonstrate that tubular epithelial cells exposed to Ang II transform from a normally quiescent epithelial phenotype to a pro-inflammatory one, which is characterized by increased ICAM-1 and LOX-1 expression, enhanced leukocyte adherence, greater epithelial permeability and apoptosis.

Ang II is a major stimulator of LOX-1 gene expression in HCAECs. LOX-1 gene expression is up-regulated both transcriptionally and translationally. NF- $\kappa \mathrm{B}$ is the major transcription factor for Ang II-induced LOX-1 promoter activation (6). LOX-1 is also found in cardiac fibroblasts and renal proximal tubules $(2,15)$. However, it is not clear whether the LOX-1 gene expression in renal tubular epithelial cells is up-regulated by Ang II. In the present study, we demonstrate that Ang II enhances LOX-1 expression, at both the gene and protein levels in HRPTEpiCs. This induction of LOX-1 expression occurs in a dose- and time-dependent fashion. However, the role of LOX-1 in the Ang II-induced dysfunction of renal tubular epithelial cells is unclear. Certain studies have reported that LOX-1 deletion reduces renal injury induced by Ang II infusion, suggesting that LOX-1 is a key modulator in the development of Ang II-induced hypertension and subsequent renal damage (10). Our study also provides evidence that LOX-1 plays an important role in Ang II-induced epithelial dysfunction in cultured HRPTEpiCs.

Evidence has shown that the Ang II-induced ICAM-1 expression and macrophage activation, as indicators of inflammation, contributed to aortic fibrosis and renal dysfunction of Ang II hypertensive rats $(5,20)$. ICAM-1 (or CD54), best known for promoting neutrophil traffic across the endothelial barrier $(21,22)$, is a specific component of LOX-1-mediated inflammatory vascular responses in vitro (23) and in vivo (24). ICAM-1 is a cell-surface protein typically stimulated by cytokines, cell stress, and oxidants $(20,25)$. Dominguez et al showed that the levels of ICAM-1 which participate in leukocyte migration and epithelial dedifferentiation, were also found to be up-regulated in the tubules of diabetic obese rats and that this alteration was associated with LOX-1 expression induction (4). In the present study, the transfection with LOX-1 siRNA was able to attenuate both the Ang II-induced ICAM-1 expression and the adhesion between the monocytes and the HRPTEpiCs.

We observed that NADPH oxidase expression was also greatly increased in the HRPTEpiCs after treatment with Ang II. This was confirmed in the present study by the examination of NADPH oxidase (the p22phox, p47phox and Nox4 subunits) expression and the direct measurement of intracellular ROS by using DCF fluorescence. The modulation of biological signaling pathways by ROS depends on the upstream ligand-dependent stimulation of ROS production and on the specific interactions of ROS with individual downstream pathways. However, NADPH oxidases are especially important due to their primary role as modulators of redox signaling (26). In the present study, we found that the inhibition of NADPH oxidase by apocynin or DPI reduced adhesion molecule expression and monocytic adherence. All of these findings strongly indicate that the ICAM-1 up-regulation is promoted by oxidative stress as a direct consequence of LOX-1 activation, and that LOX-1 mediates the oxidative and inflammatory effects of Ang II in HRPTEpiCs. The inhibition of NADPH oxidase also inhibited LOX-1 expression and the pre-treatment of the HRPTEpiCs with LOX-1 siRNA reduced the Ang II-induced up-regulation of NADPH oxidase (the p22phox, p47phox and Nox4 subunits). Thus, Ang II induces LOX-1 expression, which activates NADPH oxidase-mediated ROS generation, and which in turn up-regulates LOX-1 expression. These observations provide compelling evidence for a positive feedback loop involving Ang II, NADPH oxidase-mediated ROS generation, and LOX-1.

ROS are important mediators of apoptosis. ROS generation is often responsible for the mitochondria-mediated signaling pathway of apoptosis (27). In the present study, we found that the ROS generation was the earliest apoptotic signal, usually occurring within $15 \mathrm{~min}$ after the addition of Ang II. In addition, ROS have been shown to activate LOX-1 and the LOX-1 activation itself leads to ROS generation. Here, we also demonstrate that LOX-1 knockdown reduces ROS generation in HRPTEpiCs.

Studies have shown that oxLDL leads to endothelial apoptosis in a Bcl-2- and caspase-9-dependent manner. This pro-apoptotic effect of oxLDL can be blocked by a LOX-1 antibody and LOX-1 antisense oligonucleotides $(28,29)$. Previous studies have demonstrated that both Ang II and oxLDL stimulate intracellular oxidative stress and induce the expression of LOX-1 in endothelial cells $(30,31)$. Here, we investigate the involvement of LOX-1 in Ang II-induced oxidative stress and apoptosis. In mammalian cells, apoptotic stimuli cause cytochrome c release from the mitochondria, inducing a series of biochemical reactions, that ultimately result in caspase activation and subsequent cell death (32). Cytochrome c release is known to be regulated by $\mathrm{Bcl}-2$ family proteins, including Bcl-2 and Bcl-xL. These proteins bind to the mitochondrial outer membrane and block cytochrome c efflux (33). The results presented in this study provide evidence that Ang II induces apoptosis in HRPTEpiCs by triggering the mitochondrial apoptosis pathway, resulting in the down-regulation of the anti-apoptotic (Bcl-2) and in the up-regulation of the pro-apoptotic (Bax) proteins, the release of mitochondrial protein cytochrome $\mathrm{c}$ and the activation of caspase-3. These alterations were prevented by LOX siRNA and NADPH oxidase inhibitors.

MAPK signaling pathways are activated by multiple cellular stress signals, including oxidative stress and inflammatory cytokines, and play a vital role in tubular epithelial apoptosis, inflammation and renal injury $(2,34)$. Previous studies have shown that redox-sensitive transcription factors such as $\mathrm{NF}-\kappa \mathrm{B}$ are activated via signaling pathways that include p38 and p44/42 MAPK $(9,34)$. The LOX-1 activation 
by oxLDL and Ang II enhances oxidative stress and intense oxidative stress further stimulates MAPKs and redox-sensitive transcription factors such as NF-кB $(20,31)$. These events are followed by the the up-regulation of genes such as ICAM-1 and MCP-1, leading to cellular dysfunction, apoptosis, cell injury, monocyte adhesion and activation and eventually atherosclerosis $(34,35)$. Thus, we examined the role of redoxsensitive MAPKs in Ang II-mediated inflammation and apoptosis. HRPTEpiCs treated with Ang II did not exhibit any changes in p38 or p44/42 MAPK protein levels. However, the phosphorylation of these proteins was increased 2-3-fold. MAPK phosphorylation was blocked when the cells were treated with NADPH oxidase inhibitors or LOX-1 siRNA. On the contrary, pre-treatment with MAPK inhibitors significantly reduced ICAM-1 expression, monocytic adherence and caspase- 3 activation in the Ang II-induced HRPTEpiCs. These observations suggest that Ang II rapidly elevates ROS levels, and then multiple downstream events are activated via secondary messengers, including the phosphorylation of p38 MAPK or ERK. Both of these proteins can then cause NF- $\mathrm{BB}$ activation, enabling its nuclear translocation and subsequent regulation of pro-inflammatory gene expression $(2,36)$. The pre-treatment with MAPK inhibitors had no effect on LOX-1 expression in the Ang II-induced HRPTEpiCs, suggesting that MAPK activation is downstream of ROS generation in response to Ang II/NADPH oxidase/LOX-1 induction.

In summary, the results of our experiments indicate that Ang II enhances LOX-1 expression and oxidative stress in proximal renal tubular epithelial cells, and that the activation of the MAPK pathway occurs downstream of the Ang II/ ROS/LOX-1 cascade. Our data also demonstrate a new role for LOX-1 as a mediator of Ang II-induced oxidative functional damage, providing a new target for the treatment of renal disease.

\section{Acknowledgements}

This study was supported in part by a Traditional Chinese Medicine research grant (no. wzzsb0907) from the Department of Health of Fujian Province of China.

\section{References}

1. Zhou MS, Schuman IH, Jaimes EA and Raij L: Renoprotection by statins is linked to a decrease in renal oxidative stress, TGFbeta, and fibronectin with concomitant increase in nitric oxide bioavailability. Am J Physiol Renal Physiol 295: F53-F59, 2008.

2. Kelly KJ, Wu P, Patterson CE, Temm C and Dominguez JH: LOX-1 and inflammation: a new mechanism for renal injury in obesity and diabetes. Am J Physiol Renal Physiol 294: F1136-F1145, 2008

3. Kim HJ, Moradi H, Yuan J, Norris K and Vaziri ND: Renal mass reduction results in accumulation of lipids and dysregulation of lipid regulatory proteins in the remnant kidney. Am J Physiol Renal Physiol 296: F1297-F1306, 2009.

4. Dominguez JH, Mehta JL, Li D, et al: Anti-LOX-1 therapy in rats with diabetes and dyslipidemia: ablation of renal vascular and epithelial manifestations. Am J Physiol Renal Physiol 294: F110-F119, 2008.

5. Liao TD, Yang XP, Liu YH, et al: Role of inflammation in the development of renal damage and dysfunction in angiotensin IIinduced hypertension. Hypertension 52: 256-263, 2008.

6. Chen J, Liu Y, Liu H, Hermonat PL and Mehta JL: Molecular dissection of angiotensin II-activated human LOX-1 promoter. Arterioscler Thromb Vasc Biol 26: 1163-1168, 2006.
7. Li D, Saldeen T, Romeo F and Mehta JL: Oxidized LDL upregulates angiotensin II type 1 receptor expression in cultured human coronary artery endothelial cells: the potential role of transcription factor NF-kappaB. Circulation 102: 1970-1976, 2000.

8. Griendling KK, Minieri CA, Ollerenshaw JD and Alexander RW: Angiotensin II stimulates NADH and NADPH oxidase activity in cultured vascular smooth muscle cells. Circ Res 74: 1141-1148, 1994.

9. Pueyo ME, Gonzalez W, Nicoletti A, Savoie F, Arnal JF and Michel JB: Angiotensin II stimulates endothelial vascular cell adhesion molecule-1 via nuclear factor-kappaB activation induced by intracellular oxidative stress. Arterioscler Thromb Vasc Biol 20: 645-651, 2000.

10. Hu C, Kang BY, Megyesi J, Kaushal GP, Safirstein RL and Mehta JL: Deletion of LOX-1 attenuates renal injury following angiotensin II infusion. Kidney Int 76: 521-527, 2009.

11. Denizot F and Lang R: Rapid colorimetric assay for cell growth and survival. Modifications to the tetrazolium dye procedure giving improved sensitivity and reliability. J Immunol Methods 89: 271-277, 1986.

12. Degterev A, Boyce M and Yuan J: A decade of caspases. Oncogene 22: 8543-8567, 2003.

13. Pagano PJ, Chanock SJ, Siwik DA, Colucci WS and Clark JK: Angiotensin II induces p67phox mRNA expression and NADPH oxidase superoxide generation in rabbit aortic adventitial fibroblasts. Hypertension 32: 331-337, 1998.

14. Ha $\mathrm{H}$ and Lee HB: Reactive oxygen species amplify glucose signalling in renal cells cultured under high glucose and in diabetic kidney. Nephrology (Carlton) 10: S7-S10, 2005.

15. Hu C, Dandapat A, Sun L, Khan JA, Liu Y, Hermonat PL and Mehta JL: Regulation of TGFbeta1-mediated collagen formation by LOX-1: studies based on forced overexpression of TGFbeta1 in wild-type and lox-1 knock-out mouse cardiac fibroblasts. J Biol Chem 283: 10226-10231, 2008.

16. Goncalves AR, Fujihara CK, Mattar AL, Malheiros DM, Noronha Ide L, de Nucci G and Zatz R: Renal expression of COX-2, ANG II, and AT1 receptor in remnant kidney: strong renoprotection by therapy with losartan and a nonsteroidal antiinflammatory. Am J Physiol Renal Physiol 286: F945-F954, 2004.

17. Taniyama Y and Griendling KK: Reactive oxygen species in the vasculature: molecular and cellular mechanisms. Hypertension 42: 1075-1081, 2003.

18. Vaziri ND, Bai Y, Ni Z, Quiroz Y, Pandian R and RodriguezIturbe B: Intra-renal angiotensin II/AT1 receptor, oxidative stress, inflammation, and progressive injury in renal mass reduction. J Pharmacol Exp Ther 323: 85-93, 2007.

19. Ruiz-Ortega M, Lorenzo O, Ruperez M, Konig S, Wittig B and Egido J: Angiotensin II activates nuclear transcription factor kappaB through AT(1) and AT(2) in vascular smooth muscle cells: molecular mechanisms. Circ Res 86: 1266-1272, 2000.

20. Lin CX, Rhaleb NE, Yang XP, Liao TD, D'Ambrosio MA and Carretero OA: Prevention of aortic fibrosis by $\mathrm{N}$-acetyl-serylaspartyl-lysyl-proline in angiotensin II-induced hypertension. Am J Physiol Heart Circ Physiol 295: H1253-H1261, 2008.

21. Cernuda-Morollon E and Ridley AJ: Rho GTPases and leukocyte adhesion receptor expression and function in endothelial cells. Circ Res 98: 757-767, 2006.

22. Cook-Mills JM and Deem TL: Active participation of endothelial cells in inflammation. J Leukoc Biol 77: 487-495, 2005.

23. Li D, Chen H, Romeo F, Sawamura T, Saldeen T and Mehta JL: Statins modulate oxidized low-density lipoprotein-mediated adhesion molecule expression in human coronary artery endothelial cells: role of LOX-1. J Pharmacol Exp Ther 302: 601-605, 2002.

24. Inoue K, Arai Y, Kurihara H, Kita T and Sawamura T: Overexpression of lectin-like oxidized low-density lipoprotein receptor-1 induces intramyocardial vasculopathy in apolipoprotein E-null mice. Circ Res 97: 176-184, 2005.

25. Roebuck KA and Finnegan A: Regulation of intercellular adhesion molecule-1 (CD54) gene expression. J Leukoc Biol 66: 876-888, 1999.

26. Cai H, Griendling KK and Harrison DG: The vascular NAD(P)H oxidases as therapeutic targets in cardiovascular diseases. Trends Pharmacol Sci 24: 471-478, 2003.

27. Ou HC, Lee WJ, Lee IT, Chiu TH, Tsai KL, Lin CY and Sheu WH: Ginkgo biloba extract attenuates oxLDL-induced oxidative functional damages in endothelial cells. J Appl Physiol 106: 1674-1685, 2009. 
28. Li D and Mehta JL: Upregulation of endothelial receptor for oxidized LDL (LOX-1) by oxidized LDL and implications in apoptosis of human coronary artery endothelial cells: evidence from use of antisense LOX-1 mRNA and chemical inhibitors. Arterioscler Thromb Vasc Biol 20: 1116-1122, 2000.

29. Chen J, Mehta JL, Haider N, Zhang X, Narula J and Li D: Role of caspases in Ox-LDL-induced apoptotic cascade in human coronary artery endothelial cells. Circ Res 94: 370-376, 2004.

30. Tham DM, Martin-McNulty B, Wang YX, et al: Angiotensin II is associated with activation of NF-kappaB-mediated genes and downregulation of PPARs. Physiol Genomics 11: 21-30, 2002.

31. Mehta JL, Hu B, Chen J and Li D: Pioglitazone inhibits LOX-1 expression in human coronary artery endothelial cells by reducing intracellular superoxide radical generation. Arterioscler Thromb Vasc Biol 23: 2203-2208, 2003

32. Jiang $\mathrm{X}$ and Wang X: Cytochrome C-mediated apoptosis. Annu Rev Biochem 73: 87-106, 2004.
33. Yang J, Liu X, Bhalla K, et al: Prevention of apoptosis by Bcl-2: release of cytochrome $\mathrm{c}$ from mitochondria blocked. Science 275: 1129-1132, 1997.

34. Chao J, Li HJ, Yao YY, Shen B, Gao L, Bledsoe G and Chao L: Kinin infusion prevents renal inflammation, apoptosis, and fibrosis via inhibition of oxidative stress and mitogen-activated protein kinase activity. Hypertension 49: 490-497, 2007.

35. Mehta JL, Chen J, Hermonat PL, Romeo F and Novelli G: Lectin-like, oxidized low-density lipoprotein receptor-1 (LOX-1): a critical player in the development of atherosclerosis and related disorders. Cardiovasc Res 69: 36-45, 2006.

36. Yoshiyama M, Omura T, Takeuchi K, et al: Angiotensin blockade inhibits increased JNKs, AP-1 and NF-kappa B DNAbinding activities in myocardial infarcted rats. J Mol Cell Cardiol 33: 799-810, 2001. 